\title{
Total Anomalous Pulmonary Venous Return
}

National Cancer Institute

\section{Source}

National Cancer Institute. Total Anomalous Pulmonary Venous Return. NCI Thesaurus.

Code $C 98585$.

A rare congenital heart disorder in which all four pulmonary veins are not connected to the left atrium and drain into the systemic veins or the right atrium instead. Infants present with cyanosis, lethargy, tachypnea, respiratory infections, and poor growth. 